\title{
Acceso abierto en publicaciones científicas emergentes en Ecuador
}

\section{( J. Andrés Delgado Ron}

Universidad Tecnológica Equinoccial, Ecuador / andres.delgadoron@ute.edu.ec / http://orcid.org/oooo-0001-7051-6481

\section{Resumen}

Objetivo: Determinar el estado del acceso abierto en publicaciones científicas emergentes del Ecuador.

Metodología: Se realizó una recopilación de información en el sistema regional de información en línea para revistas científicas de América Latina, el Caribe y Portugal (Latindex) con el fin de identificar revistas científicas en línea vigentes de Ecuador; posteriormente se visitó estas páginas para compilar datos sobre derechos de autor, licencia y características de uso. Se compilaron indicadores bibliométricos para su análisis. Finalmente, se analizó la situación legal y económica del acceso abierto en Ecuador.

Conclusiones: El acceso abierto se ha vuelto una práctica común en Ecuador en los últimos años, de acuerdo a algunos indicadores y probablemente debido a un marco legal favorable. Sin embargo, el desconocimiento del tema y de metodologías para su implementación podría estar inhibiendo una práctica más amplia del acceso abierto en el país.

\footnotetext{
Abstract

Open access in emerging scientific journals in Ecuador

Aim: To determine the state of open access in emerging scientific journals from Ecuador. Methodology: A search was conducted in the regional online information system for scientific journals from Latin America, the Caribbean and Portugal (Latindex) in order to identify current online scientific journals from Ecuador. Subsequently, these pages were visited to compile data on copyright, license, and usage characteristics. Bibliometric indicators were compiled as well. Finally, the legal and economic frameworks of open access in Ecuador were analyzed. Conclusions: Open access has become a common practice in Ecuador in recent years, according to some indicators and probably due to a favorable legal framework. However, ignorance of the topic and methodologies for its implementation could be inhibiting a broader practice of open access in the country.
}

\section{Palabras clave}

Revistas científicas Acceso abierto Ecuador Latindex

Keywords

Scientific journals Open access Ecuador Latindex 


\section{Introducción}

El acceso abierto a la literatura científica se refiere a la disponibilidad gratuita en internet de artículos científicos que permiten al usuario la lectura, descarga, copia, distribución, impresión, búsqueda o enlace al texto completo de dichos artículos. Además permite rastrearlos para su indización, incorporarlos como datos en un software, o utilizarlos para cualquier otro propósito que sea legal, sin barreras financieras, legales o técnicas, aparte de las que son inseparables del acceso a internet (Chan et al., 2002). El modelo de acceso abierto inició como una reacción al modelo clásico de publicación (Gómez et al., 2009), a inicios de este siglo y adquirió fuerza a raíz de estudios que determinaron que el acceso abierto incrementa el número de usuarios potenciales por artículo, por lo cual incrementa también su uso e impacto (Harnad y Brody, 2004). En 2006 las publicaciones de acceso abierto constituían el $4.6 \%$ del total de la literatura científica a nivel mundial. En 2016, esa cifra se incrementó a $13.75 \%$ (Scimago, 2017).

Muchos de los países de América Latina han publicado declaraciones y leyes para favorecer el acceso abierto (Costa y Leite, 2016). Los países de la región pueden ser beneficiados por el acceso abierto debido a que este hace visible su investigación mejorando el flujo de información Sur-Sur y Sur-Norte (Chan y Costa, 2005). Según un estudio realizado a editores y gestores de revistas científicas en varias reuniones regionales, se estimó que un $62.87 \%$ de las publicaciones en el continente utilizan alguna modalidad de acceso abierto (Fischman et al., 2010).

Sánchez-Tarrago y colaboradores (2016) encontraron que la mayoría de editores y autores en América Latina desconocen el concepto de acceso abierto, lo cual sugiere que su uso se ve principalmente motivado por otros factores como conveniencia económica, políticas institucionales, marcos normativos o incluso las opciones por defecto ofrecidas por sistemas informáticos en los que se gestionan las revistas actualmente.

Como vemos, la temática del acceso abierto puede ser abordada desde varias perspectivas: legal, porque implica la gestión de derechos de autor y otros derechos de acceso a la información; tecnológica, pues requiere de medidas efectivas que permitan el uso y reuso de publicaciones; económica, porque implica un modelo de negocio distinto al modelo clásico de publicación que afecta tanto a autores como a lectores; y bibliométrica, pues el libre acceso a la información también incide en el impacto de los artículos publicados. En el presente trabajo, trataré de abordar cada una de ellas con la finalidad de ofrecer una perspectiva global sobre el acceso abierto en publicaciones científicas emergentes en Ecuador.

Antes de continuar, cabe aclarar que existen dos estrategias de acceso abierto. La ruta verde, que incentiva al uso de repositorios para archivar "pre-prints" o borradores de artículos científicos, antes de su revisión por pares; y la ruta dorada que fomenta la publicación de artículos en revistas científicas de acceso abierto (Costa y Leite, 2016). Esta investigación se centrará en los avance de la "ruta dorada" en Ecuador.

\section{Métodos}

Se realizó un análisis de las publicaciones digitales indexadas en el sistema regional de información en línea para revistas científicas de América Latina, el Caribe, España y Portugal (Latindex) por tratarse del sistema más exhaustivo de publicaciones científicas editadas en los países iberoamericanos (Cerda y Lara, 2011). Latindex evalúa cualitativamente el grado de cumplimiento de las revistas académicas y científicas iberoamericanas sobre presentación de la revista, características de gestión y política 
editorial y características de contenido, entre otras variables (Román Román, Vázquez Valero y Urdín Caminos, 2002).

Latindex cuenta con un "directorio" que incluye información bibliográfica sobre revistas tanto en soporte impreso como electrónico; un "catálogo" donde únicamente se incluyen las revistas que cumplen los criterios de calidad editorial de Latindex; y una sección de "revistas en línea" para aquellas que tienen una versión electrónica disponible.

Con fecha de corte 13 de octubre, se realizó una búsqueda en su sitio web, incluyendo únicamente las revistas en línea, segmentadas por país. Bajo estos criterios, Ecuador cuenta con 102 publicaciones en línea de entre 550 listadas en su directorio, un $18.5 \%$ del total. Se procedió a descargar el archivo CSV de la búsqueda inicial, la que proporcionó los campos de título, editorial, situación actual, ISSN-e y año de inicio.

Una vez depurada la base de datos se procedió a visitar los 102 registros individuales para agregar campos en cada entrada de los siguientes campos: número de características cumplidas, número de características no cumplidas, naturaleza de la organización que publica, si la revista es o no arbitrada, la URL de la revista y la naturaleza de la publicación.

Posteriormente, se verificó la validez de la URL de cada una de las revistas. En caso de que la URL se encontrara fuera de línea, se realizó una búsqueda manual para determinar que la revista no haya sido trasladada a otra dirección electrónica. Una vez determinada la URL definitiva de cada revista, se ingresó a los portales digitales de cada revista para recopilar información sobre derechos de autor y licencia en notas al pie de página, políticas de publicación, avisos de derecho de autor y/o último número disponible en línea para determinar si las revistas contenían o no un aviso de uso exclusivo de derechos de autor, si declaraba o no licencia de uso de contenidos y, de ser el caso, el tipo de licencia. Estos campos fueron añadidos posteriormente a la base de datos junto con el año de publicación del último número.

Posteriormente, se caracterizó a las revistas como tecnológicamente apropiadas o no. Para calificar como tecnológicamente apropiadas, las revistas debían contar con las características descritas por Chan et al. (2002); es decir permitir la lectura, descarga, búsqueda y copia dentro del texto. Se verificó estos parámetros en un artículo disponible en el último número de cada revista. Adicionalmente, se verificó la característica de rastreo web-que es una técnica para extraer información de las revistas de forma automatizada mediante software, considerada esencial en el acceso abierto-e indexación mediante la presencia o ausencia de la revista científica en Google Scholar, de donde se obtuvieron además los índices h (Hirsch, 2005) y g (Egghe, 2006), mediante el software Publish or Perish (Harzing y Adams, 2017). La búsqueda o "query" se realizó primero mediante ISSN. La búsqueda de ISSN no siempre era posible debido a resultados conflictivos (duplicación de registro) o a que no se contaba con el ISSN de algunas publicaciones. En estos casos se acudió a otro tipo de búsqueda que incluía el nombre de la revista y/o la URL de la revista.

Se evaluaron las barreras para el envío y/o lectura de artículos en cada una de las revistas mediante la revisión de sus políticas de publicación. Se consideró que existían cargos de procesamiento de artículos si la revista así lo declaraba. También se verificó si los lectores requerían o no que los usuarios se registren en su sistema para acceder a los artículos de investigación. Todos estos datos fueron agregados a la 
base de datos inicial. Se calcularon varias variables de distribución para las revistas. Todos los queries y sus resultados fueron almacenados y se encuentran en los anexos al artículo, junto con la base de datos utilizada en la investigación.

Finalmente, se realizó una revisión del ordenamiento jurídico ecuatoriano sobre acceso abierto en revistas científicas.

\section{Resultados}

La presentación de resultados se la realizará en dos fases. Inicialmente se presentará un análisis de caracterización de las revistas digitales en Ecuador, en el cual se describirán datos sobre uso de licencias, acceso, uso de tecnologías apropiadas e índices bibliométricos. Posteriormente se realizará un análisis del marco jurídico del acceso abierto en Ecuador así como un análisis de su modelo económico en el país.

\section{La ruta dorada del acceso abierto en Ecuador}

De 102 revistas ecuatorianas en línea registradas en el directorio de Latindex, 93 se encontraban vigentes, una (1) correspondía a un registro duplicado, siete (7) habían dejado de publicarse y una (1) tenía estado "desconocido" pero se verificó posteriormente que se encontraba operativa. De 93 revistas declaradas como "vigentes", se encontró que 14 estaban fuera de línea.

\section{Licenciamiento}

De las 80 revistas en línea que forman parte de la muestra final, el $51,25 \%$ de las revistas (41) usan licencias compatibles con el acceso abierto y $48,75 \%$ (39) no usan ningún tipo de licencia. Existe una variabilidad considerable de uso de licencias dependiendo del tipo de organización que respalda la publicación. En la Figura 1 se muestran el uso de licencias de acceso abierto según el tipo de organización. Si bien es cierto que sólo 41 de las 80 revistas en la muestra utilizan licenciamiento compatible con el acceso abierto, es importante destacar que un análisis de las revistas en operación (cuyo último número fue publicado en los dos últimos años) presenta proporciones de uso diferentes. El 68\% de estas revistas utilizan licencias compatibles con el acceso abierto.

Todas las revistas que usan algún tipo de licencia, usan licencias Creative Commons $(C C)$. Estas licencias son herramientas legales mediante las cuales los autores ceden parte de sus derechos a terceros de forma voluntaria (Creative Commons, 2015). Su uso ha sido recomendado por varias declaraciones sobre el acceso abierto pues sus tres capas permiten que (a) sean comprensible para quienes hacen uso de ellas, (b) sean legibles por máquinas (por ejemplo: servicios automatizados de indización) y (c) cuenten con una base legal aplicable en el entorno internacional. Existen seis tipos de licencias Creative Commons, las cuales difieren por permitir o no obras derivadas, usos comerciales y por requerir o no la reutilización del mismo tipo de licenciamiento (Cerda y Lara, 2011). Se considera que las licencias menos restrictivas son aquellas que permiten obras derivadas y usos comerciales. A este tipo de licenciamiento también se le conoce como obra cultural libre. De las 41 revistas que declaran licencia, 17 (41\%) utilizan una licencia de trabajos culturales libres, el resto (59\%) utilizan otro tipo de licencias Creative Commons. El tipo de licencias utilizadas en las revistas científicas en línea de Ecuador se detallan en la Tabla 1. 


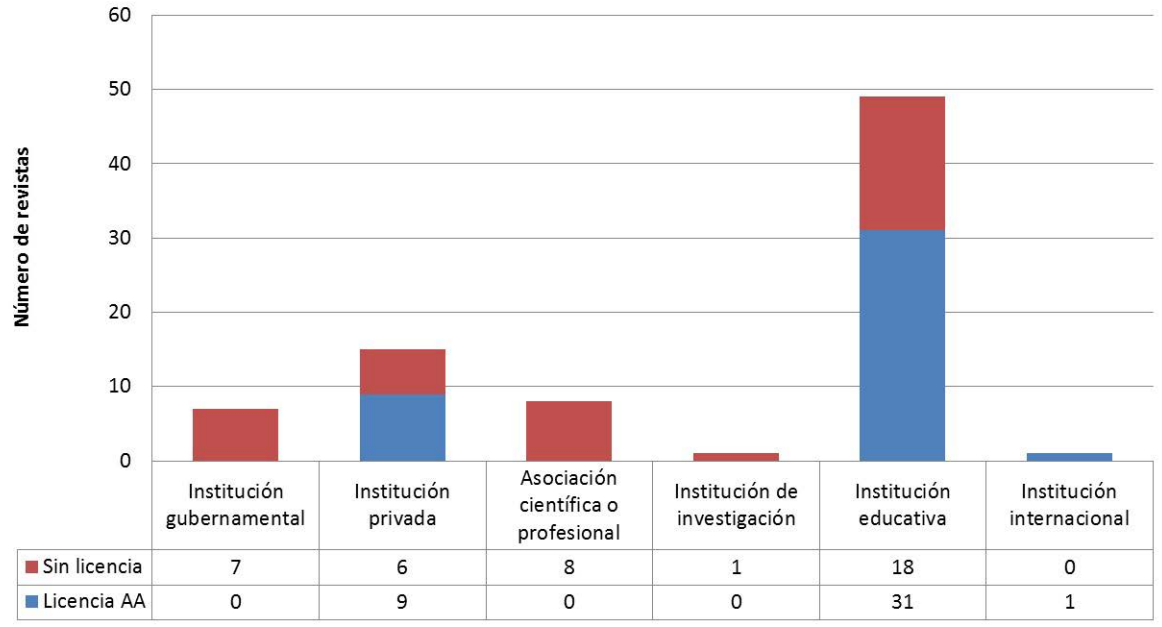

Fuente: elaboración del autor

Figura 1. Uso de licencias de acceso abierto según naturaleza de la organización

\begin{tabular}{lc}
\hline \multicolumn{2}{l}{ Licencias de las publicaciones de acceso abierto } \\
\hline CC-BY-ND & 1 \\
CC-BY-NC-ND & 9 \\
CC-BY-NC-SA & 10 \\
CC-BY-NC & 4 \\
CC-BY-SA" & 2 \\
CC-BY" & 15 \\
\hline "Trabajos culturales libres & Fuente: elaboración del autor
\end{tabular}

Tabla 1. Tipos de licencia usados

\section{Accesibilidad, rastreo e indización automática}

La mayoría de revistas en línea permiten la manipulación del texto, solo 9 de las 80 revistas no permiten buscar y copiar el texto de sus artículos. En dos casos, esto se dio porque las revistas en línea no tenían números accesibles en la web, los siete restantes se debían al formato de publicación. El 88,75\% (71) de revistas permiten la manipulación y búsqueda dentro de los documentos y el 71,25\% (57) permiten el rastreo e indización automáticas. Tres de las revistas requerían un registro previo a mostrar el texto de sus artículos (Tabla 2).

\begin{tabular}{|l|c|c|}
\cline { 2 - 3 } \multicolumn{1}{c|}{} & Licencia CC $(\mathbf{n}=\mathbf{4 1})$ & Sin Licencia $(\mathbf{n = 3 9 )}$ \\
\hline OCR & 38 & 33 \\
\hline Rastreo & 36 & 21 \\
\hline OCR + rastreo & 35 & 21 \\
\hline
\end{tabular}

Fuente: elaboración del autor 


\begin{tabular}{|c|c|c|c|c|c|c|}
\hline Título & $\begin{array}{l}\text { Número de } \\
\text { artículos }\end{array}$ & Citas & $\begin{array}{l}\text { Citas por } \\
\text { artículo }\end{array}$ & $\begin{array}{l}\text { Autores por } \\
\text { artículo }\end{array}$ & h-index & g-index \\
\hline $\begin{array}{l}\text { Revista Latinoamericana de Comunicación } \\
\text { Chasqui }\end{array}$ & 999 & 2330 & 2,33 & 1,21 & 21 & 35 \\
\hline $\begin{array}{l}\text { URVIO: Revista Latinoamericana de Estudios } \\
\text { de Seguridad }\end{array}$ & 309 & 512 & 1,66 & 1,24 & 12 & 18 \\
\hline Ciencia y Tecnología (En línea) & 96 & 120 & 1,25 & 2,79 & 6 & 9 \\
\hline $\begin{array}{l}\text { Comhumanitas: Revista Científica de } \\
\text { Comunicación }\end{array}$ & 97 & 95 & 0,98 & 1,53 & 6 & 7 \\
\hline Enfoque UTE & 200 & 173 & 0,87 & 2,62 & 6 & 9 \\
\hline Ingenius: Revista de Ciencia y Tecnología & 155 & 111 & 0,72 & 2,57 & 6 & 8 \\
\hline luris Dictio & 225 & 163 & 0,72 & 1,03 & 6 & 9 \\
\hline Revista Publicando & 852 & 175 & 0,21 & 2,16 & 6 & 10 \\
\hline $\begin{array}{l}\text { Revista Científica Interdisciplinaria: } \\
\text { Investigación y Saberes }\end{array}$ & 160 & 140 & 0,88 & 2,13 & 5 & 5 \\
\hline Revista ESPAMCIENCIA & 120 & 40 & 0,33 & 2,63 & 4 & 5 \\
\hline $\begin{array}{l}\text { Compendium: Cuadernos de Economía y } \\
\text { Administración }\end{array}$ & 41 & 18 & 0,44 & 1,85 & 3 & 3 \\
\hline Ius Humani: Revista de Derecho & 108 & 43 & 0,40 & 1,16 & 3 & 5 \\
\hline Revista Científica y Tecnológica UPSE & 219 & 47 & 0,21 & 2,35 & 3 & 4 \\
\hline $\begin{array}{l}\text { Bionatura: Revista del Centro Ecuatoriano de } \\
\text { Biotecnología y Ambiente }\end{array}$ & 42 & 9 & 0,21 & 3,19 & 2 & 2 \\
\hline Dominio de las Ciencias & 576 & 32 & 0,06 & 2 & 2 & 2 \\
\hline $\begin{array}{l}\text { Estoa: Revista de la Facultad de Arquitectura } \\
\text { y Urbanismo de la Universidad de Cuenca }\end{array}$ & 160 & 13 & 0,08 & 1,63 & 2 & 2 \\
\hline Letras Verdes & 73 & 7 & 0,10 & 1,41 & 2 & 2 \\
\hline MedPre & 59 & 9 & 0,15 & 1,88 & 2 & 2 \\
\hline Odontoinvestigación & 26 & 10 & 0,38 & 2,35 & 2 & 2 \\
\hline Polo del Conocimiento & 16 & o & 0,00 & 2,06 & 2 & 3 \\
\hline $\begin{array}{l}\text { REFCalE: Revista Electrónica Formación y } \\
\text { Calidad Educativa }\end{array}$ & 158 & 24 & 0,15 & 2,08 & 2 & 3 \\
\hline $\begin{array}{l}\text { Sophia: Colección de Filosofía de la } \\
\text { Educación }\end{array}$ & 188 & 18 & 0,10 & 1,36 & 2 & 2 \\
\hline $\begin{array}{l}\text { UNIANDES Episteme: Revista de Ciencia } \\
\text { Tecnología e Innovación }\end{array}$ & 125 & 19 & 0,15 & 2,42 & 2 & 3 \\
\hline Colloquia: Revista de Pensamiento y Cultura & 35 & 7 & 0,20 & 1 & 1 & 2 \\
\hline
\end{tabular}




\begin{tabular}{|c|c|c|c|c|c|c|}
\hline Título & $\begin{array}{l}\text { Número de } \\
\text { artículos }\end{array}$ & Citas & $\begin{array}{l}\text { Citas por } \\
\text { artículo }\end{array}$ & $\begin{array}{l}\text { Autores por } \\
\text { artículo }\end{array}$ & h-index & g-index \\
\hline Empresarial & 69 & 7 & 0,10 & 1,38 & 1 & 1 \\
\hline $\begin{array}{l}\text { Journal of Science and Research: Revista } \\
\text { Ciencia e Investigación }\end{array}$ & 55 & 2 & 0,04 & 2,13 & 1 & 1 \\
\hline $\begin{array}{l}\text { Mikarimin: Revista Científica } \\
\text { Multidisciplinaria }\end{array}$ & 68 & 4 & 0,06 & 2,46 & 1 & 1 \\
\hline $\begin{array}{l}\text { Religación: Revista de Ciencias Sociales y } \\
\text { Humanidades }\end{array}$ & 25 & 2 & 0,08 & 1,16 & 1 & 1 \\
\hline Revista Científica Ecociencia & 22 & 0 & 0,00 & 1,95 & 1 & 1 \\
\hline Revista Magazine de las Ciencias & 49 & 1 & 0,02 & 2,37 & 1 & 1 \\
\hline Tsantsa & 55 & 1 & 0,02 & 1,13 & 1 & 1 \\
\hline $\begin{array}{l}\text { Espirales : Revista Multidisciplinaria de } \\
\text { Investigación }\end{array}$ & 26 & 0 & 0,00 & 1,81 & o & o \\
\hline $\begin{array}{l}\text { Recimundo: Revista Científica Mundo de la } \\
\text { Investigación y el Conocimiento }\end{array}$ & 53 & 0 & 0,00 & 3,19 & 0 & o \\
\hline $\begin{array}{l}\text { Rehuso: Revista de Ciencias Humanísticas y } \\
\text { Sociales }\end{array}$ & 43 & o & 0,00 & 1,95 & o & o \\
\hline Revista Científica Hallazgos21 & 40 & 0 & 0,00 & 2,13 & o & o \\
\hline
\end{tabular}

Tabla 3. Indicadores bibliométricos de las revistas de acceso abierto en Ecuador

Fuente: Google Scholar y elaboración del autor

\section{Indicadores bibliométricos}

Los indicadores bibliométricos de las revistas de acceso abierto en Ecuador se presentan en la Tabla 3. En promedio, el índice-h en el subgrupo de revistas de acceso abierto es de 3,28 ( $S D \pm 3,21)$, mientras que el promedio de las revistas sin acceso abierto fue de 2,62 (SD $\pm 3,27)$. Se realizó una prueba de comparación de medias, entre ambas cifras sin encontrarse una diferencia estadísticamente significativa.

\section{Marco jurídico del acceso abierto en Ecuador}

Correa (2017) realizó un análisis jurídico sobre el acceso abierto en el país concluyendo que no existe una normativa clara al respecto, esto a pesar de que el acceso a la información pública se constituye como un derecho. No obstante, que existen al menos dos articulados legales que norman el tema de acceso abierto.

El artículo 144 de la Ley Orgánica de Educación Superior establece que "todas las instituciones de educación superior estarán obligadas a entregar las tesis (...) en formato digital para ser integradas al Sistema Nacional de Información de la Educación Superior del Ecuador" (Asamblea Nacional, 2010). Esta ley ha incentivado la creación de múltiples repositorios en las instituciones de educación superior. La Red de Repositorio de Acceso Abierto del Ecuador (RRAAE), que tiene por objetivo facilitar la gestión, descentralización, organización, preservación e interoperabilidad de los contenidos digitales de acceso abierto, ha aprovechado los recursos de estos repositorios para incrementar el acceso a trabajos de finalización de carrera (Pozo-Cabrera et al., 2016) y, hasta octubre de 2017, únicamente dos revistas científicas se encontraban integradas a su sistema.

El Código orgánico de la economía social de los conocimientos, creatividad e innovación ("Código Ingenios"), por otra parte, establece que "la información y el contenido de las bases de datos producto de las investigaciones financiadas con recursos públicos serán de acceso abierto" excepto cuando "no sea conveniente (...) por razones de seguridad, 
soberanía, protección de datos, o de actuales o futuros derechos de propiedad intelectual" (Asamblea Nacional, 2016).

Adicionalmente, el Código Ingenios establece en su artículo 114 que, en el caso de

las obras creadas en centros educativos, universidades, escuelas politécnicas, institutos superiores técnicos, tecnológicos, pedagógicos, de artes y los conservatorios superiores, e institutos públicos de investigación como resultado de su actividad académica o de investigación [incluyendo] artículos académicos, u otros análogos (...) el establecimiento tendrá una licencia gratuita, intransferible y no exclusiva para el uso no comercial de la obra con fines académicos.

Esto quiere decir que todas las instituciones arriba mencionadas son facultadas por la ley para implementar una ruta verde del acceso abierto incluso sin contar con permiso explícito del autor.

\section{Economía del acceso abierto en Ecuador}

El análisis de la economía de la producción científica de acceso abierto debe partir de la unidad productiva de la cual hace parte.

En el Sur Global, es común que la unidad productiva no sea la revista o editorial, más la institución que la financia (por ejemplo: un instituto o universidad). En este sentido, la publicación científica es un gasto añadido que es subvencionado y ni los lectores ni los autores de la revista intervienen para cubrir los cargos por procesamiento de artículos.

En el Norte Global, en cambio, las editoriales se han manejado como una unidad de negocio independiente. El gasto procedente de los cargos por procesamiento de artículos debe ser cubierto por dicha unidad de negocio mediante el cobro de dinero, sea a los usuarios siempre (modelo clásico) (Mueller-Langer y Watt, 2013), a los usuarios durante un tiempo limitado (ruta verde del acceso abierto) o a los autores y/o sus auspiciantes (ruta dorada del acceso abierto) (Harnad y Brody, 2004).

Dado que ninguna las revistas analizadas, declara el cobro de costos para procesamiento de artículos, ni costos de suscripción a los lectores, hemos de suponer que los costos de publicación han sido enteramente asumidos por las instituciones que albergan a estas revistas.

\section{Discusión}

El uso de licencias se ha vuelto cada vez más común en las revistas ecuatorianas, esto tal vez se deba al hecho de que varias de las bases de datos regionales e internacionales que incrementan la visibilidad de las publicaciones científicas exigen que las revistas sean de acceso abierto. Adicionalmente, muchas de las revistas utilizan plataformas de gestión que promueven el acceso abierto por defecto, lo cual podría explicar porque algunas de las revistas en el estudio declaran favorecer el acceso abierto sin contar con una herramienta legal adecuada que lo permita. Las instituciones auspiciantes de las revistas deben considerar los estudios de Sánchez-Tarragó et al. (2016) sobre la falta de conocimiento sobre acceso abierto en autores y editores al momento de valorar la sostenibilidad de sus políticas de acceso abierto y también deberán evaluar la utilidad de licencias para implementarlas cuando sea apropiado.

La gran mayoría de revistas en línea permiten el acceso al texto de sus artículos pero un número mucho menor están preparadas para ser rastreadas e indizadas de manera 
automática. Es importante considerar que existe un mayor nivel de exigencia técnica para implementar este tipo de características. Es probable que los nuevos sistemas automatizados de gestión de revistas, configuradas por defecto, reduzcan esta brecha significativamente en los próximos años, si las instituciones invierten en recursos apropiados (servidores) y en capacitación de personal.

Existen pocos estudios sobre acceso abierto en Ecuador. González y Pérez (2015) realizaron un análisis de indicadores bibliométricos en siete revistas ecuatorianas indexadas en el directorio de revistas de acceso abierto (DOAJ, por sus siglas en inglés) encontrando que las publicaciones tienen un impacto "bajo" en base al número de citas, lo cual concuerda con nuestros hallazgos siguiendo una metodología similar en Latindex. Sin embargo, es importante considerar que la metodología de recolección de datos puede confundir datos de las versiones impresas de las revistas con sus versiones en línea.

Otra de las limitaciones del estudio, es la falta de actualización del directorio de Latindex. En el análisis fue posible identificar revistas que no se encontraban en línea y revistas que se encontraban en línea pero que han dejado de publicar, ambos criterios de exclusión del listado de revistas en línea. Asimismo, existen varias revistas que cumplen todas las características para ser consideradas de acceso abierto pero que no se encuentran indizadas en Latindex. Para realizar una evaluación más significativa del acceso abierto en Ecuador, es necesaria una mejor coordinación entre actores del ecosistema de publicaciones-indización a nivel nacional.

En cuanto al marco legal, cabe señalar que no existe un marco específico que regule el acceso abierto en Ecuador pero sí varios articulados que lo favorecen de forma indirecta. Las instituciones de educación superior están facultadas para archivar toda su producción científica, y obligadas a publicar toda obra académica realizada con fines de obtención de grado. Asimismo, las bases de datos de investigación financiada con fondos públicos deben ser puestas a disposición de la gente. Acciones de promoción sobre estos marcos legales pueden tener un impacto significativo en la expansión del acceso abierto en el país.

Finalmente, ninguna revista declaró el cobro de cargos por procesamiento de artículos, con base en esta cifra podríamos afirmar que en la muestra seleccionada no existe la práctica de usar a las revistas como una herramienta de lucro sin calidad científica (revistas depredadoras). Sin embargo, este es un dato auto reportado en la página web de las revistas y es posible que algunas de ellas cobren a los autores sin declararlo. Desde una perspectiva opuesta, algunos críticos han sugerido que el acceso abierto, al reducir los ingresos de una revista científica, podría poner en peligro la sostenibilidad de la misma (McCabe, 2011). Sin embargo, como bien señala Litman, (2006) la economía de las publicaciones científicas debe ser analizada dentro de un contexto mayor: el presupuesto institucional de investigación y, por tanto, el análisis pertinente es si cómo el acceso abierto en las revistas ecuatorianas, afectan otros indicadores de producción científica, inciden en su prestigio, y conducen fondos hacia las instituciones.

\section{Conclusión}

El número de revistas en línea que usan licencias de acceso abierto ha incrementado durante los últimos años. De la misma manera, el marco regulatorio ecuatoriano ha cambiado para favorecer las prácticas del acceso abierto. No obstante, la falta de consistencia en algunas revistas entre su intención declarada de favorecer el acceso abierto y la ausencia de licencias o estándares que permitan un acceso abierto plenojunto con un claro modelo económico que permita una operación adecuada de las revistas-pone en tela de duda su sostenibilidad a largo plazo. 


\section{Q Referencias bibliográficas}

»Cerda, Alberto y Juan Lara. 2011. Políticas editoriales de publicaciones académicas en línea en Latinoamérica: Informe final). Santiago de Chile: ONG Derechos digitales.

»Chan, Leslie, et al. 2002. Budapest Open Access Initiative. <http://www.citeulike. org/group/15400/article/11855295> [Consulta: 24 octubre 2017].

"Chan, Leslie y Sely Costa. 2005. Participation in the global knowledge commons: challenges and opportunities for research dissemination in developing countries. En New library world. Vol. 106, no. 3/4, 141-163.

»Correa, José. 2017. Acceso abierto a la información: Garantías y retos en el Ecuador. En II Congreso Internacional Ciencia y Tecnología. <http://investigacion. utmachala.edu.ec/proceedings/index.php/utmach/article/download/173/146> [Consulta: 24 octubre 2017].

»Costa, Michelli y Fernando Leite. 2016. Open access in the world and Latin America: A review since the Budapest Open Access Initiative. En Transinformação. Vol. 28, no. 1, 33-46.

"Creative Commons. 2015. About the licenses. <http://www.citeulike.org/ group/15400/article/13913645>. [Consulta: 24 octubre 2017].

»Ecuador. Asamblea Nacional. 2010. Ley Orgánica de Educación Superior. Quito, Ecuador.

»Ecuador. Asamblea Nacional. 2016. Código Orgánico de la Economía Social de los Conocimientos, Creatividad e Innovación. Quito, Ecuador.

»Egghe, Leo. 2006. Theory and practise of the g-index. En Scientometrics. Vol. 69 , no. 1, 131-152.

》 Fischman, Gustavo; Juan Alperín y John Willinsky. 2010. Visibility and quality in Spanish-language Latin American scholarly publishing. En Information technologies \& international development. Vol. 6, no. 4, 1.

»Gómez, N.; A. Bustos-Gonzalez; J. Santillan-Aldana y O. Arias. 2009. Open access indicators and information society: the Latin American case. En OCLC Systems \& Services: International digital library perspectives. Vol. 25, no. 2, 82-92.

»González, Jorge y Yudeisy Pérez. 2015. Análisis de las revistas latinoamericanas de Acceso Abierto. El caso Ecuador. En Revista Publicando. Vol. 2, no. 2, 12-23.

" Harnad, Stevan y Tim Brody. 2004. Comparing the impact of open access (OA) vs. non-OA articles in the same journals. En D-lib Magazine. Vol. 10, no. 6.

»Harzing, Anne-Wil y Davis Adams. 2017. Publish or perish: Realising Google Scholar's potential to democratise citation analysis. <https://harzing.com/ download/gsday_madrid.pdf> [Consulta: 24 octubre 2017].

»Hirsch, Jules. 2005. An index to quantify an individual's scientific research output. En Proceedings of the National academy of Sciences of the United States of America. Vol. 102, no. 46, 16569-16572.

" Litman, Jessica. 2006. The economics of open access law publishing. En Lewis \& Clark L. Rev. Vol. 10, 779. 
» McCabe, Mark. 2011. Online access and the scientific journal market: An economist's perspective. En Vol. Draft Report for the National Academy of Sciences. 1-36.

》Mueller-Langer, Frank y Richard Watt. 2013. Optimal pricing and quality of academic journals and the ambiguous welfare effects of forced open access: A two-sided model. En RatSWD Working Paper. No. 223

»Pozo-Cabrera, Enrique, et al. 2016. Epistemología, acceso abierto e impacto de la investigación científica. Cuenca: Universidad Católica de Cuenca.

»Román Román, Adelaida; Manuela Vázquez Valero y Carmen Urdín Caminos. 2002. Los criterios de calidad editorial Latindex en el marco de la evaluación de las revistas españolas de humanidades y ciencias sociales. En Revista Española de Documentación Científica. Vol. 25, no. 3, 286-307.

"Sánchez-Tarragó, et al. 2016. Scientific journals in Latin America on their way towards open access: A diagnosis on policies and editorial strategies. En Transinformação. Vol. 28, no. 2, 159-172.

» SClmago. 2017. SJR - SClmago Journal \& Country Rank. <http://www.scimagojr. com>. [Consulta: 24 octubre 2017]. 
